\title{
Passivity analysis of discrete-time hybrid systems using piecewise polynomial storage functions
}

\author{
Alberto Bemporad, Gianni Bianchini, Filippo Brogi, Graziano Chesi
}

\begin{abstract}
This paper proposes some sufficient criteria based on the computation of polynomial and piecewise polynomial storage functions for checking passivity of discrete-time hybrid systems in piecewise affine or piecewise polynomial form. The computation of such storage functions is performed by means of convex optimization techniques via the sum of squares decomposition of multivariate polynomials.

Index Terms-Hybrid systems, passive systems, discrete-time models, sum of squares decomposition.
\end{abstract}

\section{INTRODUCTION}

Passivity is a widely adopted tool for analyzing the stability of interconnections of dynamical systems (see [23], [12], [13]) and is used in several domains of engineering sciences, such as in electrical circuit and mechanical system analysis (see [2]), and even in the study of complex phenomena (see [8]). In particular, passivity is exploited in robotics as a key concept for stability analysis of human/machine interactions involving haptic interfaces (see [15], [9], [11]).

Passivity analysis of interconnected systems hinges upon the ability of characterizing the passivity properties of each single dynamical system. For linear systems a solid theory and analytical/numerical criteria are available, and theoretical characterizations were developed for smooth nonlinear dynamical systems [13]. Although most of the passivity characterizations were proposed for continuous-time models, a few results were developed for discrete-time models [5], and recently also for sampled-data systems [21].

In many practical applications, some of the system components exhibit a heterogeneous dynamical discrete and continuous nature that cannot be captured by smooth models because of abrupt mode switches. The study of such hybrid systems, that has massively emerged in the last few years, was devoted to analyzing the dynamical interaction between continuous and discrete signals in one common framework (see [1]). Passivity analysis of hybrid models has received little attention, with the contributions of [6], [14], [24] and [18], in which notions of passivity for continuous-time hybrid systems are formulated.

In this paper we address the passivity property of discretetime hybrid systems in piecewise affine (PWA) form [20] or piecewise polynomial (PWP) form. Our motivating practical reason for addressing hybrid passivity issues in discrete-time stems from the need of studying haptic problems, where a haptic device interacts with a naturally discrete-time virtual environment (see [11], [9]). Indeed, in some typical haptic

\footnotetext{
Dipartimento di Ingegneria dell'Informazione, Università di Siena, Via Roma 56, 53100 Siena, Italy \{bemporad, giannibi\}@dii.unisi.it
}

problems the virtual environment is often modeled as a discrete-time equivalent of a suitable mechanical system (see [9]), and its interaction with the haptic device can be modeled by a hybrid discrete-time system, as briefly outlined in the example section of this paper. A possible way of ensuring the stability of the haptic loop (including the human) is to assess the passivity of the dynamics relating the applied force and the velocity of the end effector [15], [9], [11]. Therefore, a criterion for investigating the passivity of discrete-time hybrid systems is desirable.

Passivity of PWA systems employing quadratic and piecewise quadratic storage functions was addressed in [3], where several LMI-based passivity tests were developed. In this paper we propose a less conservative approach for proving the passivity of a given PWA or PWP system by means of polynomial or piecewise polynomial storage functions. Such storage functions are constructed via semidefinite programming by means of the sum of squares decomposition of multivariate polynomials [17].

Sum of squares methods for the computation of piecewise polynomial Lyapunov functions have been exploited for analyzing stability of continuous-time hybrid and switched systems [19]. In this paper we use a similar idea for passivity analysis in discrete-time, although the approach can be easily generalized to the continuous-time case.

\section{PROBLEM FORMULATION AND PRELIMINARIES}

We consider discrete-time systems of the form

$$
\begin{aligned}
x_{k+1}= & f_{i}\left(x_{k}, u_{k}\right) \\
y_{k}= & h_{i}\left(x_{k}, u_{k}\right) \\
& i \in \mathcal{I}
\end{aligned} \quad \text { if }\left[\begin{array}{l}
x_{k} \\
u_{k}
\end{array}\right] \in \chi_{i},
$$

where $k \in \mathbb{T}=\{0,1, \ldots\}, x_{k} \in \mathbb{R}^{n}$ is the state vector, $u_{k} \in \mathbb{R}^{m}$ is the system input, $y_{k} \in \mathbb{R}^{p}$ is the output vector, $f_{i}(x, u): \mathbb{R}^{n+m} \rightarrow \mathbb{R}^{n}$ and $h_{i}(x, u): \mathbb{R}^{n+m} \rightarrow \mathbb{R}^{p}$ are suitable vector fields, and $\chi_{i}, i \in \mathcal{I}$ is a partition of a given subset $\mathbb{X}$ of $\mathbb{R}^{n+m}$, being $\mathcal{I}=\{1, \ldots, s\}$. The set $\mathbb{X}$ in which the state-input pair is defined is assumed to contain the origin. Each element $\chi_{i}$ of the partition is referred to as a cell. Let each cell be defined by

$$
\chi_{i}=\left\{\left[\begin{array}{ll}
x^{T} & u^{T}
\end{array}\right]^{T} \in \mathbb{X} \quad: g_{i r}(x, u) \geq 0, \quad r=1, \ldots, r_{i}\right\}
$$

where $g_{i r}(x, u): \mathbb{R}^{n+m} \rightarrow \mathbb{R}, r=1, \ldots, r_{i}, i \in \mathcal{I}$ are suitable functions.

From the standard dissipativity notion for discrete-time systems (see [13],[5]) we have that system (1) is dissipative with respect to a given supply function $s(u, y): \mathbb{R}^{m+p} \rightarrow \mathbb{R}$ 
if there exists a nonnegative function $V(x): \mathbb{R}^{n} \rightarrow \mathbb{R}$ with $V(0)=0$, called the storage function, such that

$$
V\left(x_{k+1}\right)-V\left(x_{k}\right) \leq s\left(y_{k}, u_{k}\right) \quad \forall u_{k}, \forall k \in \mathbb{T} .
$$

For $p=m$, system (1) is termed passive if it is dissipative with respect to the supply function $s(u, y)=y^{T} u$, i.e., if there exists a storage function $V(x)$ such that

$$
V\left(x_{k+1}\right)-V\left(x_{k}\right) \leq y_{k}^{T} u_{k} \quad \forall u_{k}, \forall k \in \mathbb{T} .
$$

Note that both the definitions of dissipativity and passivity are well-posed under the standard assumption that all state-input trajectories of the system satisfy $\left[\begin{array}{ll}x_{k}^{T} & u_{k}^{T}\end{array}\right]^{T} \in \mathbb{X}$, $\forall k \in \mathbb{T}$.

The standard approach to investigating passivity of general nonlinear systems is to check inequality (4) against storage functions of prescribed structure (see [13]). In the following sections we illustrate a method for assessing the passivity property of systems of the form (1) by means of the computation of polynomial and piecewise polynomial storage functions. The proposed approach is based on the sum of squares decomposition of multivariate polynomials. It is well-known that the sum of squares decomposition provides a satisfactory relaxation for proving polynomial positivity via semidefinite programming [17]. Specifically, let $p(x)$ be a polynomial. If there exist polynomials $s_{i}(x)$, $i=1, \ldots, k$ such that

$$
p(x)=\sum_{i=1}^{k} s_{i}^{2}(x)
$$

then it clearly follows that $p(x)$ is nonnegative. The existence of such polynomials $s_{i}(x)$ can be established through a Linear Matrix Inequality (LMI) feasibility test, which is a convex optimization problem for which powerful computational tools have been developed [10], [22]. This method has been exploited in several areas of control system theory, for example to construct Lyapunov functions for proving stability of nonlinear systems [16] and uncertain systems [7]. The standard approach is to parameterize the candidate Lyapunov function $V(x)$ as

$$
V(x)=v_{0}(x)+\sum_{i=1}^{m} c_{i} v_{i}(x)
$$

where $v_{i}(x)$ are multivariate monomials or polynomials of suitable degree, and then to formulate positivity constraints involving $V(x)$ (e.g., for a discrete-time system, positive definiteness of $V(x)$ and negative definiteness of $V\left(x_{k+1}\right)$ $V\left(x_{k}\right)$ ) by imposing that suitable polynomials which depend affinely on the coefficients $c_{i}$ are sums of squares.

In the sequel, passivity conditions will be formulated in terms of inequalities based on (4). In order to employ the convex programming techniques mentioned above, such inequalities will have to be interpreted in the sum of squares sense. This implies that the following assumption will be enforced in general.
Assumption 1: The vector fields $f_{i}(x, u)$ and $h_{i}(x, u)$ in (1) and $g_{i r}(x, u)$ in (2) are multivariate polynomials in $x$ and $u$. The resulting system is then termed piecewise polynomial (PWP).

Remark 1: A very commonly employed and widely studied class of hybrid systems is the class of piecewise affine (PWA) models. Such models are of the form

$$
\begin{aligned}
& x_{k+1}=A_{i} x_{k}+B_{i} u_{k}+\phi_{i} \\
& y_{k}=C_{i} x_{k}+D_{i} u_{k}+\psi_{i} \\
& i \in \mathcal{I} \\
& \text { if }\left[\begin{array}{l}
x_{k} \\
u_{k}
\end{array}\right] \in \chi_{i} \text {, } \\
& \chi_{i}=\left\{\left[x^{T} u^{T}\right]^{T} \in \mathbb{X}: F_{i}^{x} x \geq f_{i}^{x}, F_{i}^{u} u \geq f_{i}^{u}\right\}
\end{aligned}
$$

where $F_{i}^{x}, f_{i}^{x}, F_{i}^{u}, f_{i}^{u}, i \in \mathcal{I}$ are constant matrices/vectors. Clearly, these models fall into the wider class of PWP systems introduced in (1).

The most common way to investigate stability and passivity of PWA systems is to look for piecewise quadratic Lyapunov or storage functions. In [3], piecewise quadratic storage functions have been used to assess passivity of PWA systems, yielding a set of sufficient LMI criteria which may prove to be quite conservative. It is worth to note that looking for higher order piecewise polynomial storage functions may significantly reduce conservatism of passivity tests for this class of systems, as it will be clear from the examples presented in this paper.

\section{MAIN RESULTS}

\section{A. Passivity analysis via common polynomial storage func- tions}

It is easily seen that passivity of system (1) is ensured if there exists a common storage function satisfying inequality (4) for each subsystem defined by $\left(f_{i}(x, u), h_{i}(x, u), \quad i \in\right.$ $\mathcal{I})$. Therefore, the following sufficient condition is readily obtained.

Theorem 1: Consider system (1). If there exists a polynomial $V(x)$ such that $V(0)=0$ and

$$
\begin{array}{ll}
V(x)>0 & \forall x \neq 0 \\
V\left(f_{i}(x, u)\right)-V(x)-h_{i}^{T}(x, u) u \leq 0 & \forall(x, u), \quad \forall i \in \mathcal{I}
\end{array}
$$

then system (1) is passive with storage function $V(x)$.

Note that if system (1) is PWA and $V(x)=x^{T} P x$ is quadratic, the above condition boils down to a set of LMIs derived from the application of the KYP Lemma to each of the subsystems. Nevertheless, computing a common storage function of higher degree is in general a less conservative test for affine systems. It is not difficult to single out systems for which a common quadratic storage functions cannot be found and that can be proven to be passive via a higher order $V(x)$. It is also clear that the common storage function test turns out to be particularly conservative for switched systems, since the switching mechanism is completely disregarded. 
B. Passivity analysis via piecewise polynomial storage functions

A piecewise polynomial (PWP) candidate storage function for system (1) is a function $V(x): \mathbb{X} \rightarrow \mathbb{R}$ defined as

$$
V(x)=V_{i}(x) \quad \forall\left[x^{T} u^{T}\right]^{T} \in \chi_{i}, \quad i \in \mathcal{I},
$$

where $V_{i}(x), i \in \mathcal{I}$ are polynomials.

Let us define the set of index pairs

$$
\begin{gathered}
\mathcal{S}=\left\{(i, j): \exists x \in \mathbb{R}^{n}, u, w \in \mathbb{R}^{m}:\left[x^{T} u^{T}\right]^{T} \in \chi_{i},\right. \\
\left.\left[f_{i}(x, u)^{T} w^{T}\right]^{T} \in \chi_{j}, i, j \in \mathcal{I}\right\}
\end{gathered}
$$

i.e., the set of ordered pairs of indices corresponding to all transitions from cell $\chi_{i}$ at time $k$ to cell $\chi_{j}$ at time $k+1$ which are actually allowed to occur along all possible system trajectories. The following sufficient passivity condition holds.

Theorem 2: Consider system (1). If there exist polynomials $V_{i}(x), i \in \mathcal{I}$, such that $V_{i}(0)=0$ and

$$
\begin{array}{ll}
V_{i}(x)>0 & \forall x \neq 0, \quad \forall i \in \mathcal{I} \\
V_{j}\left(f_{i}(x, u)\right)-V_{i}(x)-h_{i}^{T}(x, u) u \leq 0 & \forall(x, u), \forall(i, j) \in \mathcal{S}
\end{array}
$$

then system (1) is passive with storage function (10).

The above result easily follows from the fact that the feasibility of the sum of squares problem in (12) ensures that the dissipation inequality (4) holds for a suitable $V(x)$ of the form (10) along all system trajectories.

Remark 2: The set of allowed transitions $\mathcal{S}$ is in general quite difficult to compute for an arbitrary PWP system. Nevertheless, the above result can be applied successfully in the case of PWA systems, since the related set $\mathcal{S}$ can be computed by means of reachability analysis using linear programming (see [4]). Otherwise, a more conservative version of Theorem 2 is readily obtained by replacing $\mathcal{S}$ with the cartesian product $\mathcal{S}^{\text {all }}=\mathcal{I} \times \mathcal{I}$.

\section{Relaxed PWP passivity test}

A certain amount of conservatism can be removed from the PWP passivity test in Theorem 2 . First, we observe that each $V_{i}(x)$ need not be positive in the whole state space but only within the associated cell $\chi_{i}$. To account for this fact, by recalling the definition (2) of $\chi_{i}$, positive definiteness of each $V_{i}(x)$ can be replaced with the relaxed condition

$$
V_{i}(x)-\sum_{r=1}^{r_{i}} a_{i r}(x, u) g_{i r}(x, u)>0 \quad \forall(x, u), x \neq 0
$$

for some positive polynomials $a_{i r}(x, u)$. In fact, since

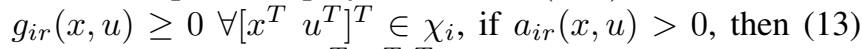
implies $V_{i}(x)>0 \forall\left[x^{T} u^{T}\right]^{T} \in \chi_{i}$.

The same reasoning can be applied to obtain a relaxation of the second inequality in (12). For all $(i, j) \in \mathcal{S}$, let us introduce the set $\tilde{\chi}_{i}^{j} \subseteq \chi_{i}$ defined as

$$
\tilde{\chi}_{i}^{j}=\left\{\left[x^{T} u^{T}\right]^{T} \in \chi_{i}: \exists w:\left[f_{i}(x, u)^{T} w^{T}\right]^{T} \in \chi_{j}\right\}
$$

i.e., the subset of state-input pairs in cell $\chi_{i}$ at time $k$ which are allowed to evolve into cell $\chi_{j}$ at time $k+1$. Suppose $\tilde{\chi}_{i}^{j}$ can be expressed in the form

$$
\tilde{\chi}_{i}^{j}=\left\{\left[x^{T} u^{T}\right]^{T} \in \mathbb{X}: g_{i j r}(x, u) \geq 0, r=1, \ldots r_{i j}\right\}
$$

where $g_{i j r}(x, u), r=1, \ldots r_{i j}$ are suitable polynomials. Then, the second inequality in (12) can be replaced by the relaxed condition

$$
\begin{aligned}
& V_{j}\left(f_{i}(x, u)\right)-V_{i}(x)-h_{i}^{T}(x, u) u \\
& \quad+\sum_{r=1}^{r_{i j}} b_{i j r}(x, u) g_{i j r}(x, u) \leq 0
\end{aligned} \quad \forall(x, u), \quad \forall(i, j) \in \mathcal{S}
$$

for some positive polynomials $b_{i j r}(x, u)$. Summing up, we have the following result.

Theorem 3: Consider system (1). If there exist polynomials $V_{i}(x), i \in \mathcal{I}, a_{i r}(x, u)>0, r=1, \ldots, r_{i}, i \in \mathcal{I}$ and $b_{i j r}(x, u)>0, r=1, \ldots, r_{i j},(i, j) \in \mathcal{S}$ such that $V_{i}(0)=0$ and

$$
\begin{array}{cl}
V_{i}(x)-\sum_{r=1}^{r_{i}} a_{i r}(x, u) g_{i r}(x, u)>0 & \forall(x, u), x \neq 0 \\
V_{j}\left(f_{i}(x, u)\right)-V_{i}(x)-h_{i}^{T}(x, u) u & \forall(x, u), \forall(i, j) \in \mathcal{S} \\
\quad+\sum_{r=1}^{r_{i j}} b_{i j r}(x, u) g_{i j r}(x, u) \leq 0 &
\end{array}
$$

then system (1) is passive with storage function (10).

Remark 3: Unfortunately, a parameterization of the sets $\tilde{\chi}_{i}^{j}$ of the form (15) seldom exists or can be computed easily. Nevertheless, for some important classes of systems such as PWA models this task is readily accomplished. Consider the PWA model defined by (7),(8). The set $\tilde{\chi}_{i}^{j}$ turns out to be the polytope

$$
\begin{aligned}
& \tilde{\chi}_{i}^{j}= \\
& \left\{\begin{array}{ll}
{\left[x^{T}\right.} & \left.u^{T}\right]^{T} \\
F_{i}^{x} x \geq f_{i}^{x}, & F_{i}^{u} u \geq f_{i}^{u}, F_{j}^{x}\left(A_{i} x+B_{i} u+\phi_{i}\right) \geq f_{j}^{x}
\end{array}\right\}
\end{aligned}
$$

which can be expressed in the form (15).

When a parameterization of the sets $\tilde{\chi}_{i}^{j}$ of the form (15) is not available, then a more conservative result can be obtained by relaxing the second inequality in (12) with respect to the condition $\left[x^{T} u^{T}\right]^{T} \in \chi_{i} \supseteq \tilde{\chi}_{i}^{j}$ in place of $\left[x^{T} u^{T}\right]^{T} \in \tilde{\chi}_{i}^{j}$.

Corollary 1: Consider system (1). If there exist polynomials $V_{i}(x), i \in \mathcal{I}$ and $a_{i r}(x, u)>0, r=1, \ldots, r_{i}, i \in \mathcal{I}$ such that $V_{i}(0)=0$ and

$$
\begin{array}{ll}
V_{i}(x)-\sum_{r=1}^{r_{i}} a_{i r}(x, u) g_{i r}(x, u)>0 & \forall(x, u), x \neq 0 \\
V_{j}\left(f_{i}(x, u)\right)-V_{i}(x)-h_{i}^{T}(x, u) u & \forall(x, u), \quad \forall(i, j) \in \mathcal{S} \\
+\sum_{r=1}^{r_{i}} b_{i r}(x, u) g_{i r}(x, u) \leq 0 &
\end{array}
$$

then system (1) is passive with storage function (10).

\section{EXAMPLES}

Example 1: Consider the piecewise linear system with state vector $x^{T}=\left[\begin{array}{ll}x_{1} & x_{2}\end{array}\right]^{T}$, scalar input $u$ and scalar output 


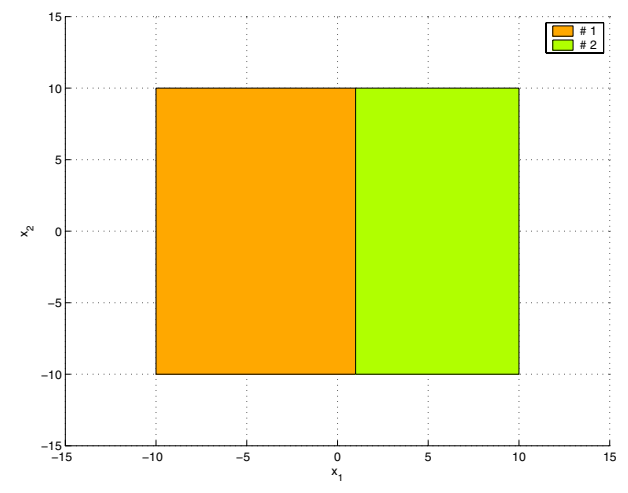

Fig. 1. Example 1: state space partition

$y$ of the form (1) described by two subsystems defined by

$$
\begin{aligned}
& f_{1}(x, u)=\left[\begin{array}{c}
0.5 x_{1}+0.4899 x_{2}+0.5477 u \\
0.9 x_{2}+0.8944 u
\end{array}\right] \\
& g_{1}(x, u)=\left[\begin{array}{c}
-0.5477 x_{1}+0.8944 x_{2}+3 u
\end{array}\right] \\
& f_{2}(x, u)=\left[\begin{array}{c}
0.6 x_{1}+0.4290 x_{2}+0.6325 u \\
0.96 x_{2}+0.6782 u
\end{array}\right] \\
& g_{2}(x, u)=\left[0.6325 x_{1}+0.6782 x_{2}+u\right]
\end{aligned}
$$

and the state space partition

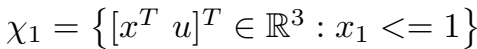

$$
\begin{aligned}
& \chi_{2}=\left\{\left[\begin{array}{ll}
x^{T} & u
\end{array}\right]^{T} \in \mathbb{R}^{3}: x_{1}>1\right\}
\end{aligned}
$$

(see Fig. 1). The set of allowed transitions turns out to be $\mathcal{S}=\mathcal{I} \times \mathcal{I}$.

For this system, neither a common quadratic nor a common quartic storage function that prove passivity is found. By means of the relaxed passivity test in Theorem 3, not even a piecewise quadratic storage function is found. Indeed, a piecewise quartic storage function can be computed as

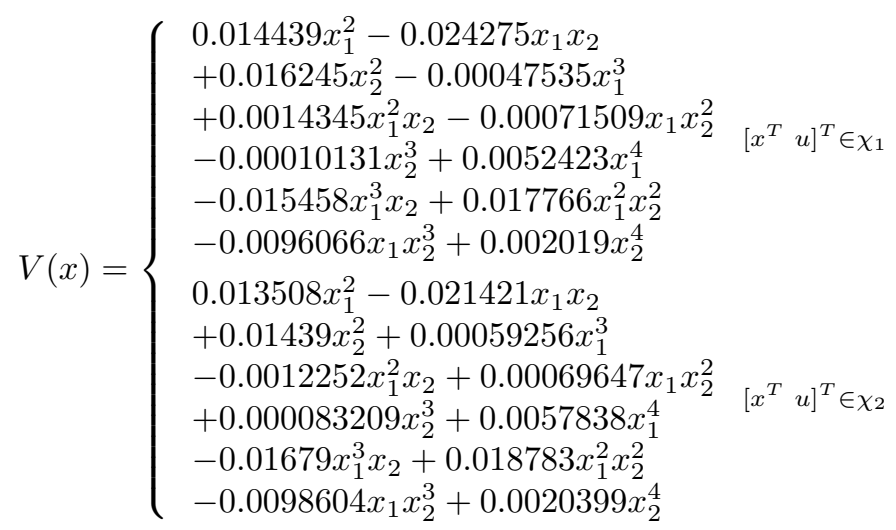

Example 2: Consider the simple haptic model in Fig. 2. The idea of modelling the interaction between the haptic device and the virtual environment with a sampled-data equivalent of a spring-mass-damper system is quite standard (see [9]). Clearly, the virtual environment is a computersimulated model and hence a pure discrete-time system. On

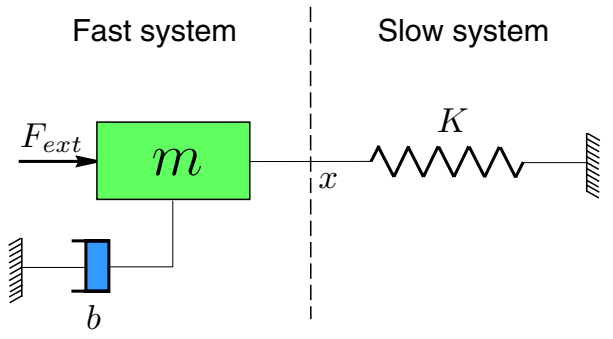

Fig. 2. Haptic model

the contrary, the haptic device dynamics is continuous-time. A possible approach to analyzing passivity of the overall model, where the input $u$ is the external force $F_{\text {ext }}$ applied to the haptic device and the output $y$ is the velocity $\dot{x}$ of the end effector, is to derive a discrete-time multi-rate model in which different sampling times $n T_{s}$ and $T_{s}(n>1)$ are used for the simulated environment and the haptic device dynamics, respectively. Let the virtual environment (slow system) be the discrete-time equivalent (with sampling time $n T_{s}$ ) of a spring system with stiffness $K$ and the haptic device dynamics (fast system) be the discrete-time equivalent (with sampling time $T_{s}$ ) of a mass $m$ and damping $b$. In order to analyze the passivity property in discrete-time, the usual $\mathrm{ZOH}$ equivalent is not suitable and it is necessary to employ the method described in [3] which preserves the passivity property of linear systems under discretization. The overall multirate system can be modeled using a discrete-time PWA description. Due to space limitations, the details are omitted here and we refer the interested reader to http://www. dii.unisi.it/ hybrid/passivity. The two affine subsystems defining the PWA model are reported in Table I, where all constants are suitable functions of the physical parameters $K, m, b$ and of the sampling time. The related state space partition is reported in Table II and is depicted in Fig. 3. The input $u$ is the external force and the output $y$ is the mass velocity. For the sake of clarity, the set $\mathcal{S}$ is expressed as the one-step reachability matrix shown in Table III.

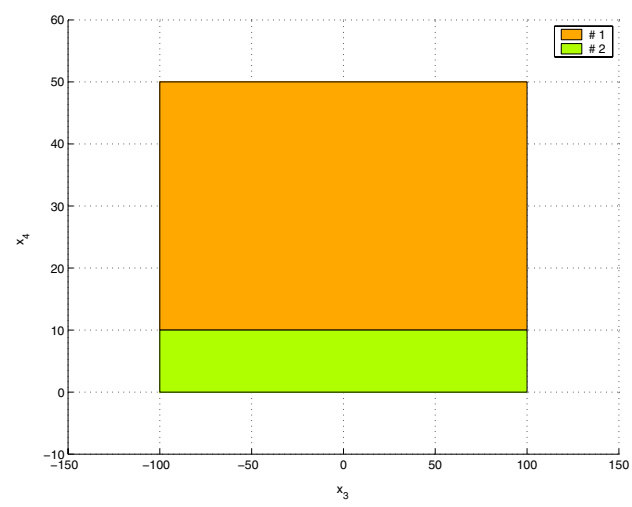

Fig. 3. Example 2: state space partition

The parameter values used for conducting passivity anal- 
TABLE I

EXAMPLE 2: PWA MODEL

\begin{tabular}{c|ccc}
\hline Cell & System Matrices \\
\hline \hline \multirow{1}{*}{$\chi_{1}$} & $A_{1}=\left[\begin{array}{cccc}a_{d_{11}} & a_{d_{12}}-K b_{d_{1}} & 0 \\
a_{d_{21}} & a_{d_{22}}-K b_{d_{2}} & 0 \\
1 & 0 & 0 & 0 \\
0 & 0 & 0 & 0\end{array}\right]$ & $B_{1}=\left[\begin{array}{l}b_{d_{1}} \\
b_{d_{2}} \\
0 \\
0\end{array}\right]$ & $\phi_{1}=\left[\begin{array}{l}0 \\
0 \\
0 \\
1\end{array}\right]$ \\
& $C_{1}=\left[\begin{array}{llll}c_{d_{1}} & c_{d_{2}}-K d_{d} & 0\end{array}\right]$ & $D_{1}=d_{d}$ & $\psi_{1}=0$ \\
\hline \multirow{1}{*}{$\chi_{2}$} & $A_{2}=\left[\begin{array}{cccc}a_{d_{11}} & a_{d_{12}}-K b_{d_{1}} & 0 \\
a_{d_{21}} & a_{d_{22}}-K b_{d_{2}} & 0 \\
0 & 0 & 1 & 0 \\
0 & 0 & 0 & 1\end{array}\right]$ & $B_{2}=\left[\begin{array}{c}b_{d_{1}} \\
b_{d_{2}} \\
0 \\
0\end{array}\right]$ & $\phi_{2}=\left[\begin{array}{l}0 \\
0 \\
0 \\
1\end{array}\right]$ \\
& $C_{2}=\left[\begin{array}{llll}c_{d_{1}} & c_{d_{2}}-K d_{d} & 0\end{array}\right]$ & $D_{2}=d_{d}$ & $\psi_{2}=0$ \\
\hline
\end{tabular}

TABLE II

EXAMPLE 2: STATE SPACE PARTITION

\begin{tabular}{l|l} 
Cell & \\
\hline \hline$\chi_{1}$ & $x_{4} \geq n$ \\
\hline$\chi_{2}$ & $x_{4}<n$ \\
\hline \hline
\end{tabular}

ysis of the model are shown in Table IV. For these values, a piecewise quartic storage function proving the passivity of the system is computed via the relaxed test in Theorem 3. The same test fails if a piecewise quadratic storage function is looked for.

Example 3: Consider a haptic model similar to the one in the previous example, in which an additional coulombian friction term with coefficient $c$ acts on the end effector, i.e.,

$$
F_{c}=c \operatorname{sgn}(\dot{x})
$$

(Fig. 4). The overall multirate system is described by the PWA model in Table V. The related state space partition is reported in Table VI and depicted in Fig. 5. The one-step reachability matrix is shown in Table VII.

With the parameter values shown in Table VIII, a piecewise quartic storage function which proves the passivity of the system is computed via the relaxed test in Theorem 3 . Also in this case, no piecewise quadratic storage function can be computed.

TABLE III

EXAMPLE 2: ONE STEP REACHABILITY MATRIX

\begin{tabular}{c||c|c} 
& may reach $\chi_{1}$ & may reach $\chi_{2}$ \\
\hline \hline$\chi_{1}$ & no & yes \\
\hline$\chi_{2}$ & yes & yes \\
\hline
\end{tabular}

TABLE IV

EXAMPLE 2: PARAMETER VALUES

\begin{tabular}{c|c} 
parameter & value \\
\hline \hline$m$ & $\mathbf{1}$ \\
\hline$b$ & $\mathbf{5}$ \\
\hline$K$ & $\mathbf{3}$ \\
\hline$T_{s}$ & $\mathbf{1 0}^{-4}$ \\
\hline$n$ & $\mathbf{1 0}$
\end{tabular}

TABLE V

EXAMPLE 3: PWA MODEL

\begin{tabular}{|c|c|c|c|}
\hline Cell & \multicolumn{3}{|c|}{ System matrices } \\
\hline$\chi_{1}$ & $\begin{array}{c}A_{1}=\left[\begin{array}{cccc}a_{d_{11}} & a_{d_{12}} & -K b_{d_{1}} & 0 \\
a_{d_{21}} & a_{d_{22}} & -K b_{d_{2}} & 0 \\
1 & 0 & 0 & 0 \\
0 & 0 & 0 & 0\end{array}\right] \\
C_{1}=\left[\begin{array}{llll}c_{d_{1}} & c_{d_{2}} & -K d_{d} & 0\end{array}\right]\end{array}$ & $\begin{array}{c}B_{1}=\left[\begin{array}{c}b_{d_{1}} \\
b_{d_{2}} \\
0 \\
0\end{array}\right] \\
D_{1}=d_{d}\end{array}$ & $\begin{array}{c}\phi_{1}=\left[\begin{array}{c}-b_{d_{1}} c \\
-b_{d_{2}} c \\
0 \\
1\end{array}\right] \\
\psi_{1}=-d_{d} c\end{array}$ \\
\hline$\chi_{2}$ & $\begin{array}{c}A_{2}=\left[\begin{array}{cccc}a_{d_{11}} & a_{d_{12}} & -K b_{d_{1}} & 0 \\
a_{d_{21}} & a_{d_{22}} & -K b_{d_{2}} & 0 \\
0 & 0 & 1 & 0 \\
0 & 0 & 0 & 1\end{array}\right] \\
C_{2}=\left[\begin{array}{llll}c_{d_{1}} & c_{d_{2}} & -K d_{d} & 0\end{array}\right]\end{array}$ & $\begin{array}{c}B_{2}=\left[\begin{array}{c}b_{d_{1}} \\
b_{d_{2}} \\
0 \\
0\end{array}\right] \\
D_{2}=d_{d}\end{array}$ & $\begin{array}{c}\phi_{2}=\left[\begin{array}{c}-b_{d_{1}} c \\
-b_{d_{2}} c \\
0 \\
1\end{array}\right] \\
\psi_{2}=-d_{d} c\end{array}$ \\
\hline$\chi_{3}$ & $\begin{array}{r}A_{3}=\left[\begin{array}{cccc}a_{d_{11}} & a_{d_{12}} & -K b_{d_{1}} & 0 \\
a_{d_{21}} & a_{d_{22}} & -K b_{d_{2}} & 0 \\
1 & 0 & 0 & 0 \\
0 & 0 & 0 & 0\end{array}\right] \\
C_{3}=\left[\begin{array}{llll}c_{d_{1}} & c_{d_{2}} & -K d_{d} & 0\end{array}\right]\end{array}$ & $\begin{array}{c}B_{3}=\left[\begin{array}{c}b_{d_{1}} \\
b_{d_{2}} \\
0 \\
0\end{array}\right] \\
D_{3}=d_{d}\end{array}$ & $\begin{array}{c}\phi_{3}=\left[\begin{array}{c}b_{d_{1}} c \\
b_{d_{2}} c \\
0 \\
1\end{array}\right] \\
\psi_{3}=d_{d} c\end{array}$ \\
\hline$\chi_{4}$ & $\begin{array}{c}A_{4}=\left[\begin{array}{cccc}a_{d_{11}} & a_{d_{12}} & -K b_{d_{1}} & 0 \\
a_{d_{21}} & a_{d_{22}} & -K b_{d_{2}} & 0 \\
0 & 0 & 1 & 0 \\
0 & 0 & 0 & 1\end{array}\right] \\
C_{4}=\left[\begin{array}{llll}c_{d_{1}} & c_{d_{2}} & -K d_{d} & 0\end{array}\right]\end{array}$ & $\begin{array}{c}B_{4}=\left[\begin{array}{c}b_{d_{1}} \\
b_{d_{2}} \\
0 \\
0\end{array}\right] \\
D_{4}=d_{d}\end{array}$ & $\begin{array}{c}\phi_{4}=\left[\begin{array}{c}b_{d_{1}} c \\
b_{d_{2}} c \\
0 \\
1\end{array}\right] \\
\psi_{4}=d_{d} c\end{array}$ \\
\hline
\end{tabular}

TABLE VI

EXAMPLE 3: STATE SPACE PARTITION

\begin{tabular}{l|l} 
Cell & \\
\hline \hline$\chi_{1}$ & $x_{2} \geq 0 \bigwedge x_{4} \geq n$ \\
\hline$\chi_{2}$ & $x_{2} \geq 0 \bigwedge x_{4}<n$ \\
\hline$\chi_{3}$ & $x_{2}<0 \bigwedge x_{4} \geq n$ \\
\hline$\chi_{4}$ & $x_{2}<0 \bigwedge x_{4}<n$ \\
\hline \hline
\end{tabular}

TABLE VII

EXAMPLE 3: ONE STEP REACHABILITY MATRIX

\begin{tabular}{c||c|c|c|c} 
& m. reach $\chi_{1}$ & m. reach $\chi_{2}$ & m. reach $\chi_{3}$ & m. reach $\chi_{4}$ \\
\hline \hline$\chi_{1}$ & no & yes & no & yes \\
\hline$\chi_{2}$ & yes & yes & yes & yes \\
\hline$\chi_{3}$ & no & yes & no & yes \\
\hline$\chi_{4}$ & yes & yes & yes & yes \\
\hline
\end{tabular}

TABLE VIII

EXAMPLE 3: PARAMETER VALUES

\begin{tabular}{c|c} 
parameter & value \\
\hline \hline$m$ & $\mathbf{1}$ \\
\hline$b$ & $\mathbf{5}$ \\
\hline$c$ & $\mathbf{0 . 2}$ \\
\hline$K$ & $\mathbf{4}$ \\
\hline$T_{s}$ & $\mathbf{1 0}^{-4}$ \\
\hline$n$ & $\mathbf{1 0}$
\end{tabular}




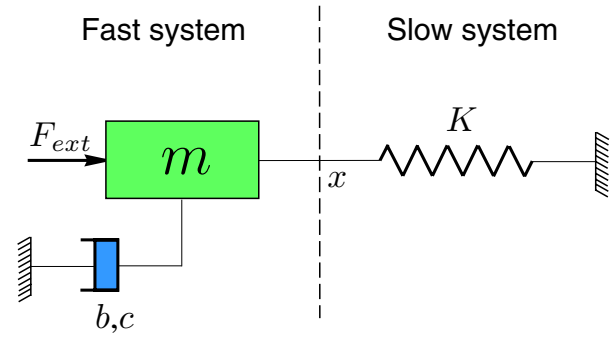

Fig. 4. Example 3: haptic model with coulombian friction

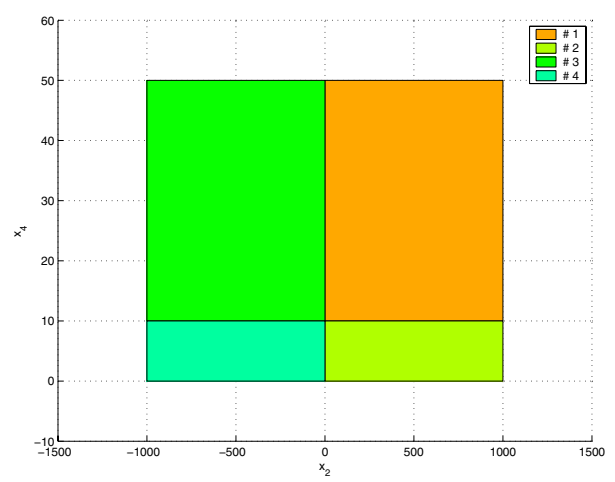

Fig. 5. Example 3: state space partition

\section{CONCLUSION}

This paper has proposed sufficient passivity analysis criteria for discrete-time hybrid systems in piecewise affine or piecewise polynomial form. Such criteria are based on computing piecewise polynomial storage functions by exploiting sum of squares decomposition techniques and semidefinite programming. The proposed approach appears particularly encouraging in the analysis and design of haptic systems, which is a subject of current investigation by the authors.

\section{REFERENCES}

[1] P.J. Antsaklis. A brief introduction to the theory and applications of hybrid systems. Proc. IEEE, Special Issue on Hybrid Systems: Theory and Applications, 88(7):879-886, July 2000.

[2] S. Arimoto. Control Theory of Non-Linear Mechanical Systems: A Passivity-Based and Circuit-Theoretic Approach, volume 49. Oxford Engineering Science, Oxford, UK, 1996.

[3] A. Bemporad, G. Bianchini, F. Barbagli, and F. Brogi. Passivity analysis and passification of discrete-time hybrid systems. In IFAC World Congress, Praha, CZ, 2005.

[4] A. Bemporad, F. Torrisi, and M. Morari. Optimization-based verifi cation and stability characterization of piecewise affi ne and hybrid systems. In Proceedings of the 3rd International Workshop on Hybrid Systems, pages 504-509, Pittsburgh, USA, 2000. Lecture notes in computer science, Springer Verlag.

[5] C. Byrnes and W. Lin. Losslessness, feedback equivalence, and the global stabilization of discrete-time nonlinear systems. IEEE Transactions on Automatic Control, 39(1):83-98, 1994.

[6] M.K. Camlibel, W.P.M.H. Heemels, and J.M. Schumacher. On linear passive complementarity systems. European Journal of Control, 8(3), 2002.

[7] G. Chesi, A. Garulli, A. Tesi, and A. Vicino. Parameter-dependent homogeneous Lyapunov functions for robust stability of linear timevarying systems. In 43rd IEEE Conf. on Decision and Control, pages 4095-4100, Paradise Island, Bahamas, 2004.
[8] L. O. Chua. Passivity and complexity. IEEE Transactions on Circuits and Systems-I, 46(1):71-82, 1999.

[9] J.E. Colgate and G.G. Schenkel. Passivity of a class of sampled-data systems: application to haptic interfaces. Journal of robotic systems, 14(1):37-47, 1997.

[10] P. Gahinet, A. Nemirovski, A. J. Laub, and M. Chilali. LMI Control Toolbox for use with Matlab. The Math Works Inc.: User's Guide, 1995.

[11] B. Hannaford and J.H. Ryu. Time domain passivity control of haptic interface. IEEE Transactions on Robotics and Automation, 18:1-10, 2002.

[12] D. J. Hill and P. J. Moylan. Connections between fi nite-gain and asymptotic stability. IEEE Transactions on Automatic Control, 25(5):931-936, 1980

[13] R. Lozano, B. Brogliato, O. Egeland, and B. Maschke. Dissipative systems analysis and control. Springer Verlag, 2000.

[14] S. Mahapatra. Stability of Hybrid Haptic Systems. PhD thesis, University of Illinois, Chicago, Illinois, 2003.

[15] B. E. Miller, J. E. Colgate, and R.A. Freeman. Guaranteed stability of haptic systems with nonlinear virtual environments. IEEE Transactions on Robotics and Automation, 16(6):712-719, 2000.

[16] A. Papachristodoulou and S. Prajna. On the construction of Lyapunov functions using the sum of squares decomposition. In Proc. of 41 st IEEE Conf. on Decision and Control, pages 3482-3487, Las Vegas, Nevada, 2002.

[17] P. A. Parrilo. Structured semidefinite programs and semialgebraic geometry methods in robustness and optimization. Ph. D. thesis, California Institute of Technology, Pasadena, CA, USA, 2000.

[18] A. Pogromski, M. Jirstrand, and P. Spangeus. On stability and passivity of a class of hybrid systems. In Proceedings of the 37th IEEE Conference on Decision and Control, pages 3705-3710, Tampa, Florida, USA, 1998.

[19] S. Prajna and A. Papachristodoulou. Analysis of switched and hybrid systems - beyond piecewise quadratic methods. In IEEE American Control Conference, Denver, CO, USA, 2003.

[20] E.D. Sontag. Nonlinear regulation: The piecewise linear approach IEEE Transactions on Automatic Control, 26(2):346-358, April 1981.

[21] S. Stramigioli, C. Secchi, A. J. van der Schaft, and C. Fantuzzi. A novel theory for sampled data system passivity. In IEEE/RSJ Int. Conf. on Intelligent Robots and System, volume 2, pages 1936-1941, Lausanne, Switzerland, 2002.

[22] J. F. Sturm. Using SeDuMi 1.02, a MATLAB toolbox for optimization over symmetric cones. Optimization Methods and Software, 1112:625-653, 1999.

[23] J. C. Willems. Dissipative dynamical systems - Part I: General theory. Arch. Rational Mech. Anal., 45:321-351, 1972.

[24] M. Zefran, F. Bullo, and M. Stein. A notion of passivity for hybrid systems. In IEEE Conference on Decision and Control, pages 768 773, Orlando, FL, 2001. 